\title{
Producción de alimentos y desbalances macroeconómicos
}

\author{
Roberto Rivera Campos
}

La acumulación de las últimas décadas en los países latinoamericanos se ha desarrollado frecuentemente a la par de intensos procesos inflacionarios y/o elevados déficits de la balanza de pagos. No hay duda que la articulación de estos fenómenos constituye uno de los obstáculos fundamentales que cuestionan la viabilidad de los modelos vigentes y la creatividad de los conductores de la economía para mantener en el fuluro ritmos satisfactorios de crecimiento estable, en vista de las exigencias que los actuales niveles de pobreza de la región imponen a los procesos de acumulación.

La acumulación de capital en una economia no planificada ex ante tiende a crear desbalances intersectoriales que se van corrigiendo automáticamente a través de los distintos mecanismos de ajuste, uno de los cuales es el nivel de precios. Los esquemas de reproducción de Max (1976) permitieron comprender cómo estos desequilibrios intersectoriales que provoca la acumulación de capital ponen en acción mecanismos de recuperación del balance macroeconómico de los sistemas productivos. Asimismo, los esquemas de reproducción de Kalecki (1983) y su hipótesis de formación de precios diferenciada por sectores, nos han mostrado las raices del impulso inflacionario que proviene de la agricultura cuando ésta se rezaga en su crecimiento respecto de los restantes sectores. Este último fenómeno fue capturado por Noyola (1984) para conceptualizarlo como presión inflacionaria básica de origen agrícola y articularlo con otros elementos conceptualizados como mecanismos de propagación para construir, de esta manera, la base de la interpretación estructuralista de la inflación.

Precisar la articulación entre la acumulación de capital, la inflación y 
la balanza de pagos posee la mayor relevancia práctica en estos momentos en que se debate sobre los estilos de desarrollo alternativos para las regiones subdesarrolladas. El objeto de este documento es, entonces, presentar una formalización de la noción de la presión inflacionaria básica de origen agrícola en un contexto de acumulación de capital en una economía abierta. Para ello, en la primera sección se presenta una síntesis de la perspectiva estructuralista; en la segunda, recurriendo a los esquemas de reproducción de Kalecki y adoptando su distinción sectorial de formación de precios, se elabora un modelo que capta a nuestro entender lo que constituye una presión inflacionaria básica; en la tercera sección, se determina la trayectoria de acumulación balanceada no inflacionaria y se analizan las implicaciones del crecimiento no balanceado para la cuenta externa y para los precios internos. En la cuarta sección se ilustra el análisis con alguna evidencia empírica en el caso salvadoreño. Finalmente se presentan algunas conclusiones.

\section{La perspectiva estructuralista de la Inflación}

El primer planteamiento estructuralista articulado sobre la naturaleza de la inflación latinoamericana se encuentra en el trabajo de Juan Noyola: "El desarrollo económico y la inflación en México y otros paises latinoamericanos", de 1956. Partiremos de este trabajo porque, a nuestro entender, aqui se encuentra la estructura análitica básica sobre la cual se levantaron los trabajos estructurales posteriores acerca de este fenómeno.

Según Noyola, "la inflación no es un fenómeno monetario, (sino) el resultado de desequilibrios de carácter real que se manifiestan en forma de aumentos del nivel de precios, (los cuales son) mucho más perceptibles en los paises subdesarrollados que en los paises industriales ${ }^{* 1}$.

Para entender este carácter real de la inflación hay que introducir al análisis, señala el autor, "una serie de elementos derivados de la observación de la estructura y del funcionamiento de la economia de nuestros paises". ${ }^{2}$ Noyola distingue tres tipos diferentes de elementos: los de carácter estructural, los de carácter dinámico y los de carácter institucional. Entre los primeros, el autor identifica la distribución de la población por ocupaciones y las diferencias de productividad entre los sectores económicos; entre los segundos, las diferencias en los ritmos de crecimiento entre el conjunto de la economia y algunos sectores específicos; y entre los últimos, el grado de monopolio de la economia, los métodos de fijación de los precios, el grado de organización sindical, la organización y el funcionamiento del Estado, y el grado y orientación de su interven- 
ción en la vida económica.

A partir de estos distintos elementos, el autor estructura un modelo inflacionario, no formalizado, en que se distinguen dos categorias fundamentales. Por un lado, las presiones inflacionarias básicas; de las cuales depende primordialmente la intensidad de inflación, y que se originan comunmente en desequilibrios localizados casi siempre en dos sectores: el comercio exterior y la agricultura; y por otro, los mecanismos de propagación, que inciden secundariamente, entre los cuales Noyola distingue los siguientes: el mecanismo fiscal, el mecanismo de crédito y el mecanismo de reajuste de precios e ingresos.

Veamos sintéticamente cómo se articulan estos elementos en la perspectiva estructuralista. Comencemos con las presiones inflacionarias básicas. Si el sector agricola se rezaga con respecto al crecimiento del resto de la economia, la oferta agricola no acompanará el crecimiento de la demanda, resultante del crecimiento del empleo y de los insumos agricolas en el resto de la economía, con lo cual surgirá una presión inflacionaria por exceso de demanda agrícola, es decir, una presión inflacionaria básica de origen agricola. Por otro lado, si el sector exportador no crece al mismo ritmo que las importaciones, impulsadas estas últimas por el crecimiento del resto de la economia, surgirán dificultades para importar; en estas circunstancias aparecerán presiones inflacionarias internas a través de dos vías: la primera, por las devaluaciones provocadas por los déficits externos, las cuales impulsarán los precios hacia arriba a través de los mayores costos en moneda nacional de los insumos importados; y la segunda, por la necesidad de sustituir importaciones bajo condiciones de mayores costos de producción internos debido, entre otras cosas, a la escasez de recursos naturales, a las limitaciones del mercado y a la baja productividad industrial. Se tratará pues, de una presión inflacionaria básica originada en el sector externo.

Estos dos tipos de presiones inflacionarias básicas pueden irradiarse o amortiguarse dentro del resto de la economia, según sean sus características estructurales e institucionales especíticas, a través de los mecanismos de propagación. Así, por ejemplo, la política fiscal de tributación y de subsidios puede aumentar o disminuir los costos $y$, en consecuencia, expandir o amortiguar una presión inflacionaria básica. Asimismo, la expansión del crédito bancario hacia el sector privado y hacia el sector público para financiar el déficit fiscal, puede provocar presiones adicionales de demanda sobre sectores económicos de lento crecimiento o sobre recursos escasos. Finalmente, el mecanismo de ajuste de precios e ingresos puede impulsar aún más la inflación. Su efectividad dependerá de factores institucionales, tales como la organización sindical y el grado 
de monopolio de la industria, es decir, de su grado de oligopolización. Del primero dependerá la capacidad de los trabajadores de proteger sus ingresos reales frente a la inflación ${ }^{3}$; del segundo, la capacidad de los empresarios para transferir los incrementos de costos a los precios y de proteger los márgenes de ganancias trente a la inflación 4 .

La sintesis que hemos presentado del modelo estructural de la inflación nos permite apreciar que se trata de una combinación de acciones de elementos de demanda y de costos sobre los precios de las mercancias, en un contexto de especificidades de la estructura de la economía subdesarrollada. En efecto, por un lado, la presión inflacionaria que proviene del mayor crecimiento del resto de la economia respecto al sector agrícola se origina por un exceso de demanda; asimismo, la expansión del crédito como mecanismo propagador de la inflación se efectiviza mediante los excesos de demanda que provoca. Por otro lado, la presión inflacionaria básica proveniente del sector externo y los mecanismos de propagación fiscal y de ajuste de precios e ingresos se realizan a través de los costos y de los márgenes de ganancia.

\section{Acumulación e inflación: un modelo formal}

El lector de Kalecki encontrará en el modelo anterior la influencia considerable de la perspectiva de este autor. Después de rechazar por muy simplificadas los enfoques teóricos keynesiano y sueco de la inflación, Noyola se refiere en la introdución a su trabajo a la existencia de enfoques alternativos más ricos:

"Hay sin duda enfoques más refinados que arrojan mucha luz sobre la

verdadera naturaleza de la inflación, entre estos cabe citar, como todos ustedes conocen, el análisis de Kalecki, que destaca la importancia de la rigidez de la oferta y del grado de monopolio en el sistema económico" 5.

En seguida señala que tales enfoques a su vez están limitados, si no incorporan los elementos específicos de las estructuras y del funcionamiento de las economías latinoamericanas.

A continuación, siguiendo una estructura kaleckiana, se desarrollará un modelo formal sin gobierno, el cual capta las presiones inflacionarias básicas de origen agricola tal como las ha presentado el modelo estructuralista. El modelo se constituye a partir de dos elementos kaleckianos: el primero, sus esquemas de reproducción; el segundo, su distinsión entre precios determinados por demanda en la agricultura y precios determinados por costos en la manufactura ${ }^{6}$. En este trabajo se ha puesto todo el énfasis en el papel inflacionario de los alimentos agrícolas y se 
suponen dados los precios de los bienes no agrícolas, para ello se ha supuesto que la industria no utiliza insumos de la agricultura. En otro trabajo, Rivera C. (1988), se ha analizado el papel de los costos en la inflación salvadorefia.

En los esquemas de reprodución estructurados en términos nominales distinguimos cinco departamentos: producción de bienes de inversión, poco desarrollado en las economías latinoamericanas pequeñas pero existente, donde se incluye también el sector construcción; producción de bienes de consumo asalariado, que para aislar teóricamente el fenómeno bajo estudio suponemos que provienen en su totalidad de la agricultura; producción de bienes de consumo capitalista, que por la misma razón suponemos que provienen de la industria; producción de bienes de exportación, cuyos precios se tijan internacionalmente; y producción externa de bienes de importación, donde suponemos, a fin de destacar el problema agricola, que se trata de importación de alimentos para los asalariados y de consumo capitalista.

En muchos paises latinoamericanos pequeños la producción de alimentos agrícolas se desarrolla en pequenas parcelas, por arrendatarios que producen para el autoconsumo y para comerciar el excedente. Este excedente en gran parte es captado a través de diversos mecanismos por los diversos intermediarios apropiándose, generalmente, de las ganancias que resultan de los aumentos de sus precios. Este hecho debe tenerse en cuenta en la incorrecta distinción que hacemos en este departamento entre salarios y ganancias.

Así, suponiendo ausencia de gobiernos tendriamos el siguiente esquema de reproducción en términos nominales:

\begin{tabular}{|c|c|c|c|c|c|}
\hline Dep I & Dep II & Dep III & Dep IV & Total & Dep V \\
\hline $\mathrm{G}_{1}$ & $\mathrm{G}_{2}$ & $\mathrm{G}_{3}$ & $\mathrm{G}_{4}$ & $\mathbf{G}$ & ---- \\
\hline$w_{1}$ & $W_{2}$ & $W_{3}$ & $W_{4}$ & W & $\cdots$ \\
\hline IPi & CkPk & CwPw & $X P x$ & Y & $(\mathrm{Mw}+\mathrm{Mk}) \mathrm{Pr}$ \\
\hline
\end{tabular}

donde:

$\mathrm{Gi}=$ Monto de ganancias capitalistas en el departamento $\mathrm{i}$.

Wi = Monto de salarios pagados en el departamento $\mathrm{i}$.

IPi = Monto nominal del valor agregado en el departamento $\mathrm{I}$.

CkPk = Monto nominal del valor agregado en el departamento II.

$\mathrm{CWPW}=$ Monto nominal del valor agregado en el departamento III. 
XPX = Monto nominal del valor agregado en el departamento IV de bienes de exportación.

(Mw+Mk) Pm = Monto del valor de las importaciones de alimentos para los asalariados y de bienes de consumo capitalista.

Asumiendo que los asalariados no ahorran, el balance intersectorial requiere:

$$
G_{3}+M w P m=W_{1}+W_{2}+W_{4}
$$

es decir, que lo que se produce en el departamento III que no es consumido por los propios trabajadores de ese departamento completado con las importaciones de alimentos, debe igualar al consumo asalariado restante.

Sumando $\left(G_{1}+G_{2}+G_{4}\right)$ a ambos miembros de (1), agrupando y ordenando se obtiene:

$$
G=I P i+C k P k+X P x-M w P m
$$

Si definimos a $w_{1}$ como el coeficiente de participación de los salarios en el valor nominal agregado del departamento $i$, obtenemos:

$$
\begin{aligned}
& W_{1}=w_{1} I P i \\
& W_{2}=W_{2} C k P k \\
& W_{4}=W_{4} X P x
\end{aligned}
$$

Del departamento III sabemos, además, que:

$$
C W P W=G_{3}+W_{3}
$$

Sustituyendo 1, 2, 3, 4 y 5 en 6 y despejando Pw se obtiene:

$P W=\left(\frac{W_{1}}{1-W_{3}}\right) P i\left(\frac{1}{C W}\right)+\left(\frac{w_{2}}{1-W_{3}}\right) P k\left(\frac{C k}{C W}\right)+\left(\frac{W_{4}}{1-W_{3}}\right) P x\left(\frac{X}{C W}\right)-\left(\frac{1}{1-W_{3}}\right) P m\left(\frac{M W}{C m}\right)$

Este resultado nos muestra dos aspectos importantes de la problemática latinoamericana: la inflación de origen agrícola y los déficits de la balanza de pagos.

El primero de ellos, destacado por Noyola, nos dice que dados los precios internacionales, el monto de las importaciones de alimentos y los 
costos y, en consecuencia, los precios de los bienes no salariales, el nivel de precios de los alimentos agricolas crecerá cada vez que los restantes sectores de la economía crezcan más rápidamente que el sector productor de bienes agricolas de alimentación, representado ésta en la ecuación 7 por el crecimiento de cada uno de los coeficientes en paréntesis.

El segundo aspecto importante que se desprende de la ecuación 7 , no destacado suficientemente en el artículo de Noyola, es que el resto de la economía puede crecer por encima de la producción interna de bienes salarios y aún asi mantener precios estables, siempre que se tenga la capacidad de aumentar las importaciones - el término

$\left(\frac{P m}{1-w_{3}} \cdot \frac{M w}{C w}\right)-\begin{aligned} & \text { o que esté dispuesto a soportar déficits en la balan- } \\ & \text { za de pagos. }\end{aligned}$

La historia reciente de la industrialización y de la urbanización acelerada de América Latina ha estado invariablemente asociada al desarrollo de grandes presiones inflacionarias proveniente del estancamiento de su producción agrícola de alimentación. Estas presiones inflacionarias, resultado de nuestros procesos de acumulación, han desaparecido en algunos períodos de nuestra historia, tal es el caso de la estabilidad de precios de la economía centroamericana durante los años sesenta, período en el cual, a pesar de la rápida acumulación de la industria, la captación de divisas provenientes de los buenos precios del café y de las inversiones extranjeras resultado de la expansión del Mercado Común Centroamericano, permitió expandir las importaciones para complementar la oferta doméstica de alimentos.

\section{El balance no inflacionario de la acumulación}

Aislemos el impacto que la acumulación provoca en los precios agrícolas de alimentación a través de la demanda, suponiendo inicialmente que la producción para exportación y los volumenes de importación de alimentos permanecen constantes. Debe observarse que en el modelo que estamos analizando el crecimiento del sector inversión aumenta la demanda de bienes agricolas a través de dos mecanismos, uno directo y otro indirecto. El primero es el resultado del aumento del volumen de salarios pagados en el sector productor de bienes de inversión. El segundo es más complejo. El aumento en la producción del sector de bienes de inversión no sólo aumenta en este mismo sector el volumen de salarios, sino también el volumen de ganancias y, en consecuencia, aumenta la demanda de bienes de consumo capitalista producidos en el sector II. Si el sector industrial II reacciona ante este crecimiento de la demanda 
aumentando su nivel de capacidad utilizada y no aumentando los precios, to cual es el resultado de suponer la existencia de capacidad ociosa y de fijación de precios industriales por costos, la produción de bienes de consumo capitalista aumentará y así ocurrirá también con el volumen de salarios de este sector, creando demanda adicional de bienes agrícolas de alimentación.

Asumamos la siguiente función de comportamiento del consumo capitalista.

$$
\begin{aligned}
& \text { PkCk }=\alpha G \\
& \text { PmMk }=B G
\end{aligned}
$$

es decir, que el consumo capitalista de bienes producidos internamente y de bienes importados depende del monto de ganancias con propensiones al consumo $\alpha$ y $\beta$ menores que la unidad.

Sustituyendo 2 en 8 y ordenando se obtiene en la función de consumo capitalista de bienes domésticos:

$$
C_{k}=\frac{\alpha}{(1-\alpha) P k} \quad(I P i+\bar{X} P x-\bar{M} w P m)
$$

Dividiendo ambos extremos por $\mathrm{Cw}$ y sustituyendo el resultado en 7 , se llega a la siguiente expresión de los precios agricolas:

$$
\begin{aligned}
& P w=\left(\frac{w_{1}}{1-w_{3}} P i+\frac{w_{2}}{1-w_{3}} \frac{P k \alpha P i}{(1-\alpha) P k}\right)\left(\frac{I}{C w}\right)+\left(\frac{w_{4}}{1-w_{3}} P x+\frac{w_{2}}{1-w_{3}} \frac{P k \alpha P x}{(1-\alpha) P k}\right) \\
& \left(\frac{\bar{X}}{C w}\right)-\left(\frac{P m}{1-w_{3}}+\frac{W_{2}}{1-w_{3}} \frac{P k \alpha P m}{(1-\alpha) P k}\right)\left(\frac{\bar{M} w}{C w}\right)
\end{aligned}
$$

Cada coeficiente de proporcionalidad está constituido por dos componentes: el primero corresponde al efecto directo y el segudo al efecto indirecto a través de las ganancias de los crecimientos de la inversión, de las exportaciones y de las importaciones de alimentos.

LLamemos a los primeros "a" y a los segundos "b". Tendremos entonces:

$$
P w=(a i+b i)\left(\frac{1}{C w}\right)+(a x+b x)\left(\frac{\bar{x}}{C w}\right)-(a m+b m)\left(\frac{\bar{M} w}{C w}\right)
$$


diferenciando el nivel de los precios y ordenando se obtiene:

$d P w=(a i+b i) \quad d\left(\frac{1}{C w}\right)-[(a x+b x) \bar{x}-(a m+b m) \overline{M w}] \frac{d C w}{C w^{2}}$

La acumulación balanceada, no inflacionaria, en el departamento productor de bienes de inversión se alcanzaria cuando dPw sea cero, entonces:

$$
\text { (ai+bi) } d\left(\frac{1}{C w}\right)-[(a x+b x) \bar{x}-(a m+b m) \bar{M} w] \frac{d C w}{C w^{2}}=0
$$

Resolviendo esta ecuación diferencial por integración directa, se obtiene la trayectoria de acumulación balanceada.

$$
\frac{1}{C w}=-\frac{(a x+b x) \bar{X}-(a m+b m) \bar{M} w}{(a i+b i)} \frac{1}{C w}+k
$$

donde $\mathrm{K}$ es la constante de integración cuyo valor se puede determinar si suponemos que en las condiciones iniciales la economía se encontraba sobre la trayectoria de acumulación balanceada, es decir en acumulación no inflacionaria.

En las condiciones iniciales antes definidas se tiene:

$$
K_{(0)}=\frac{10}{C w_{0}}+\frac{(a x+b x) \bar{x}-(a m+b m) M w}{a i+b i} \frac{1}{C w_{0}}
$$

en donde el índice "o" sirve para indicar el valor de una variable en las condiciones iniciales. Realizando las sustituciones necesarias se muestra que:

$$
K_{(0)}=\frac{P w_{0}}{(a i+b i)}
$$

En consecuencia, 13 se convierte en:

$$
\frac{1}{C w}=-\frac{(a x+b x) \bar{x}-(a m+b m) \overline{M w}}{(a i+b i)} \frac{1}{C w}+\frac{P w_{0}}{(a i+b i)}
$$

La ecuación 14 es la expresión matemática de la trayectoria de acumulación balanceada no inflacionaria y constituye una curva que conver- 


$$
\frac{P w_{0}}{a i+b i}
$$

Puesto que $\bar{X}$ es el volumen de exportaciones totales que sirven para financiar no sólo la importación de bienes alimenticios de consumo asalariado sino también de bienes de consumo capitalista, es plausible suponer que $(a x+b x) \bar{X}>(a m+b m) \bar{M} w$, con lo cual la pendiente de la curva sería positiva, como muestra del gráfico 1.

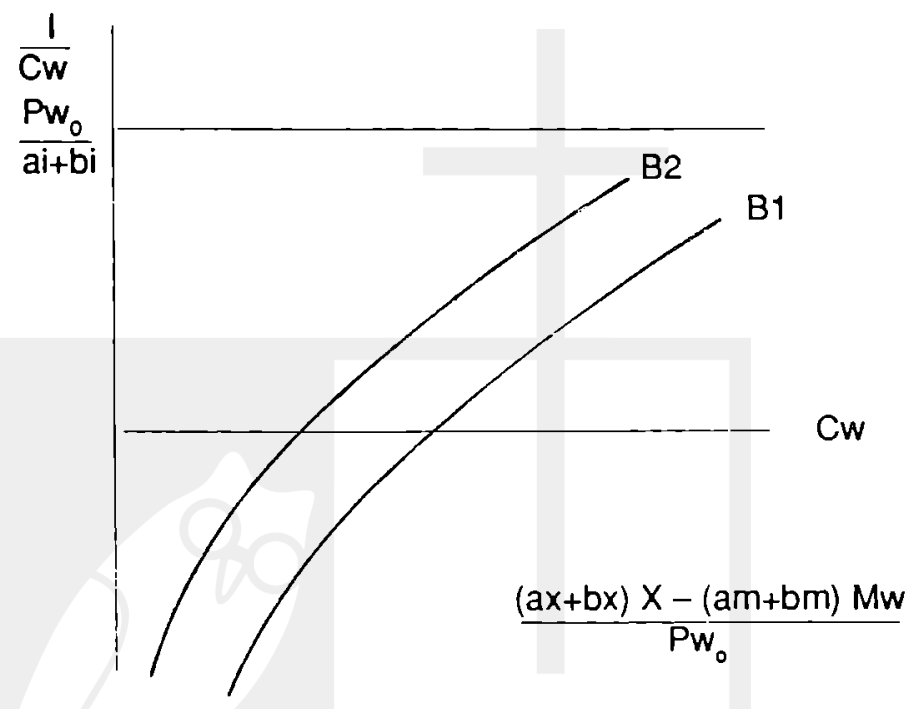

Varios elementos se distinguen en el gráfico:

1. Si la proporción de bienes de inversión respecto a la de bienes de alimentación agricolas se encuentra por encima de la trayectoria del balance $B_{1}$, se generará inflación.

2. Entre mayor es el nivel de producción agricola, el nivel de balance de la acumulación en los restantes sectores crece más que proporcionalmente. Esto significa que la transformación de la estructura productiva en favor de los sectores no agrícolas no puede realizarse si no se aumenta la producción agrícola misma. Esta relación está dada por la pendiente de la curva:

$$
\left(\frac{(a x+b x) x-(a m+b m) M w}{a i+b i}\right)\left(\frac{1}{C w^{2}}\right)>0
$$

Este aspecto nos muestra que el planeamiento de la industrializa- 
ción no se puede dar sin el planeamiento adecuado de la producción agrícola, a riesgo de provocar inflación o déficit de balanza de pagos. Un hecho que no se tomó en cuenta en la constitución del Mercado Común centroamericano.

3. Los aumentos salariales no agrícolas desplazan la trayectoria hacia la derecha con lo cual dejan expuesta la economia a mayores presiones inflacionarias para los mismos niveles de acumulación, debido a la mayor demanda de bienes salario.

4. Entre mayor es la propensión al consumo capitalista de bienes producidos internamente, más expuesta está la economia a presiones inflacionarias. Esto significa que la mayor importación de bienes manufacturados también contribuye a atenuar la presión inflacionaria de origen agrícola.

5. La presión inflacionaria que resulta de la acumulación por encima del nivel de balance puede eliminarse con mayores niveles de importaciones de alimentos, desplazándose la curva de balance hacia arriba como se muestra con la curva $\mathrm{B}_{2}$, con lo cual el control de la inflación da paso a presiones en la balanza de pagos.

La acumulación de capital no inflacionaria expande las importaciones de bienes alimentarios $(\mathrm{MW})$ y las importaciones de bienes de consumo capitalista (Mk), pues el crecimiento del sector de bienes de inversión aumenta las ganancias de los capitalistas y con ello su consumo, el cual se reparte entre bienes de producción doméstica y bienes importados con propensiones $\alpha$ y $\beta$ respectivamente.

Dado el nivel de producción doméstica de bienes alimenticios, $\mathrm{Cw}^{*}$, la función de importaciones de bienes alimenticios se obtiene a partir de la ecuación de acumulación no inflacionaria. Ordenando 14 se obtiene:

$$
M w=\frac{a x+b x}{a m+b m} \bar{x}-\frac{P w_{o}}{a m+b m} C w^{*}+\frac{a i+b i}{a m+b m} 1
$$

Combinando $2,9,10$, y 15 y simplificando algebraicamente, resulta la función de importaciones de bienes de consumo capitalista:

$$
M k=\frac{B}{I-\alpha}\left[\left(\frac{P x}{P m}-\frac{a x+b x}{a m+b m}\right) \bar{x}+\frac{P w_{o}}{a m+b m} C w^{*}\right]+\frac{B}{I-\alpha}\left(\frac{P i}{P m}-\frac{a i+b i}{a m+b m}\right) I
$$

Definiendo el déficit de la balanza comercial T de la siguiente manera:

$$
T=M w+M k-\bar{X}
$$


y sustituyendo15 y 16 se obtiene:

$$
\begin{aligned}
& T=\left[\left(1-\frac{\beta}{1-\alpha}\right)\left(\frac{a x+b x}{a m+b m}\right)+\frac{B}{1-\alpha} \frac{P x}{P m}-1\right] \bar{x}-\left[1-\frac{\beta}{1-\alpha} \frac{P w_{0}}{a m+b m} C w^{*}\right] \\
& +\left[\left(1-\frac{B}{1-\alpha}\right)\left(\frac{a i+b i}{a m+b m}\right)+\frac{\beta}{1-\alpha} \frac{P i}{P m}\right] C w^{*}\left(\frac{1}{C w^{*}}\right)
\end{aligned}
$$

Los cuadrantes del centro y de la derecha de la gráfica 2 nos muestran la operación de la ecuación. Fijado el nivel de producción de bienes de salarios, $\mathrm{Cw}^{*}$, digamos por límites estructurales, un aumento de la acumulación en otros sectores, representado en este caso el crecimiento del coeficiente $\mathrm{l} / \mathrm{CW}^{*}$, aumentará el nivel de precios de los bienes salarios a menos que se aumente el valor de las importaciones $y$, en consecuencia, el déficit en la balanza de pagos en el monto necesario para que la curva de balance no inflacionario se desplace hacia arriba. Este monto de déficit en la balanza de pagos se calcula con la siguiente expresión:

$$
\Delta T=\left[\left(1-\frac{B}{1-\alpha}\right)\left(\frac{a i+b i}{a m+b m}\right)+\frac{B}{1-\alpha} \frac{P i}{P m}\right]
$$

El mecanismo recién descrito mantendrá la estabilidad de precios hasta un punto determinado por las condiciones del sector externo. Si definimos este límite de déficit de balanza de pagos por $\mathrm{T}^{*}$ y la tasa de acumulación correspondiente por ( $/ / \mathrm{CW})$," los excesos de acumulación más allá de este punto se traducirán en incrementos de los precios de los bienes salarios tal como muestra el cuadrante izquierdo del gráfico 2.

La ecuación de precios correspondiente a la superación del límite de balanza de pagos está dada por la ecuación:

$$
P w=\left(\frac{(a x+b x) \bar{X}-(a m+b m) \bar{M} w}{C w^{*}}\right)+(a i+b i)\left(\frac{1}{C w^{*}}\right)
$$

\section{La evidencia en la economía salvadorefía}

El comportamiento del precio de alimentos, en particular del frijol, durante el primer semestre del año pasado nos ha mostrado el papel significativo de las presiones inflacionarias básicas de origen agricola en una economía como la salvadorena. Estas presiones son, como ya hemos visto, de origen estructural, no obstante se acentúan o se amortiguan por elementos conyunturales. 
En lo que sigue trataremos de presentar alguna evidencia de este carácter estructural y coyuntural de los desbalances de origen agrícola en el caso salvadorefio. La limitada información nos ha obligado a observar no el conjunto de los alimentos de origen agricola, sino a seleccionar uno de ellos. Hemos escogido el frijol para presentar la evidencia mencionada, no sólo porque en él se desarrollan con bastante nitidez los aspectos que deseamos mostrar, sino también porque el frijol constituye el segundo componente principal, después del maíz, en la dieta básica de la mayoría de los salvadoreños de bajos ingresos, como se muestra a continuación.

\section{Gráfica 2}

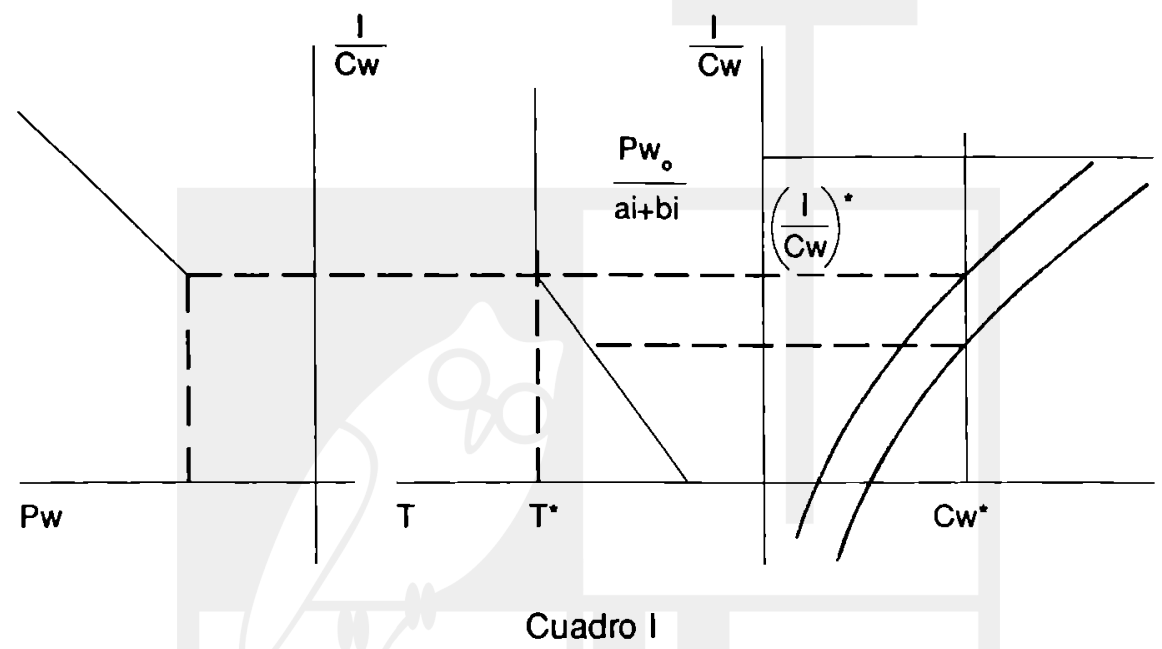

Estructura del gasto en alimentos por estrato de ingreso 1976-1977. (\%)

\begin{tabular}{|c|c|c|c|c|c|}
\hline & \multicolumn{3}{|c|}{ Grupos de ingresos ( $\notin$ mes/hogar) } & \multirow[b]{2}{*}{$\begin{array}{c}\text { De } \\
600 / 999\end{array}$} & \multirow[b]{2}{*}{$\begin{array}{l}\text { Más de } \\
1000\end{array}$} \\
\hline $\begin{array}{l}\text { Menos } \\
\text { de } 100\end{array}$ & $\begin{array}{c}\mathrm{De} \\
100 / 199\end{array}$ & $\begin{array}{c}\text { De } \\
200 / 299\end{array}$ & $\begin{array}{c}\mathrm{De} \\
300 / 599\end{array}$ & & \\
\hline 30.0 & 31.7 & 23.6 & 19.5 & 12.5 & 10.3 \\
\hline $\begin{array}{r}12.9 \\
4.4\end{array}$ & $\begin{array}{r}11.8 \\
5.2\end{array}$ & $\begin{array}{l}9.6 \\
5.0\end{array}$ & $\begin{array}{l}7.8 \\
5.0\end{array}$ & $\begin{array}{l}4.9 \\
3.5\end{array}$ & $\begin{array}{l}4.9 \\
3.6\end{array}$ \\
\hline 100.0 & 100.0 & 100.0 & 100.0 & 100.0 & 100.0 \\
\hline
\end{tabular}

Fuente: calculado sobre la base del cuadro 2-1 de Llort y Trigueros (1988).

El gráfico 3 es una ilustración del comportamiento de largo plazo de los precios del trijol, de las presiones de acumulación en el resto de la economía y del papel compensador de las importaciones. Los datos de 
los precios del trijol y de sus importaciones en quintales se han iomado del cuadro 3-4 de Llori y Trigueros (1988) y los datos del PIB a precios constantes de 1962 de las Revistas Trimestrales del Banco Central de Reserva. Tanto el PIB como las importaciones netas se expresan por unidad de frijol producida (ver la ecuación de nuestro modelo). EI PIB, pues, nos sirve para simular la presión de demanda que resulta de la acumulación de capital. Por último, para volver más fácil la comparación cada variable se ha fijado en la unidad en 1962.

En la evolución de estas variables se evidencia la existencia de dos periodos con características perfectamente diferenciales. El primero, el cual se extiende hasta 1968 y que se caracteriza por una satisfactoria estabilidad de los precios del frijol. En este período, hubo estabilidad de precios aunque la acumulación industrial que facilitó el Mercado Común Centroamericano estuvo presionando excesivamente la demanda del frijol; este punto se aprecia en el gráfico en el ascenso por arriba de la unidad del coeficiente PIB/frijol. Como el mismo gráfico lo demuestra, la estabilidad mencionada fue el resultado de la correcta complementación de la insuficiente oferta interna a través de importaciones.

\section{Gráfico 3}

Acumulación, inflación y balance externo

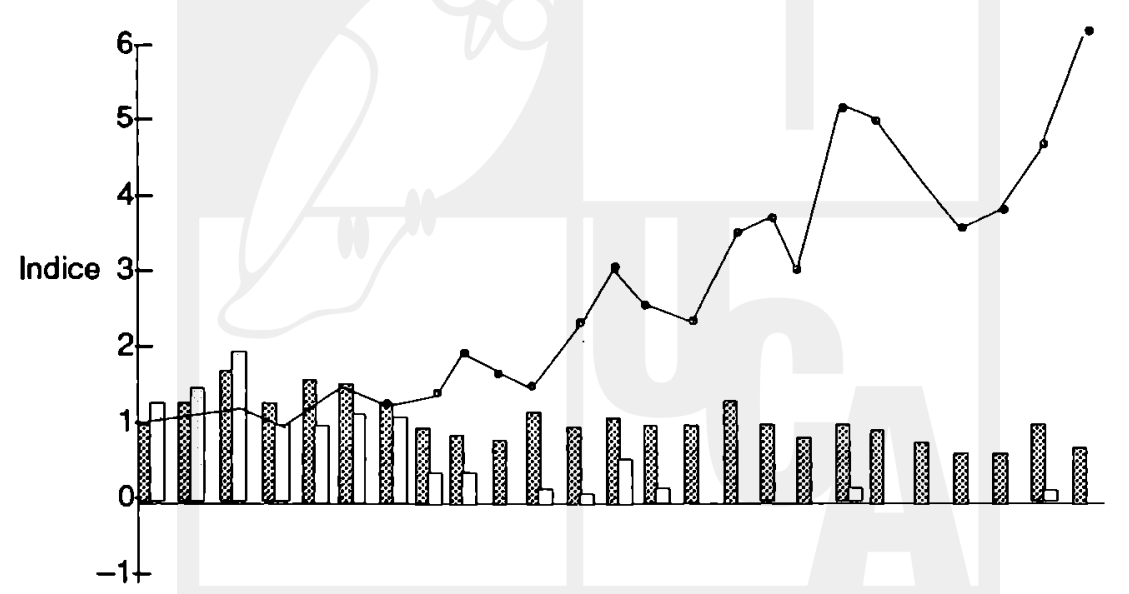

62636465666768697071727374757677787980818283848586 Años

— Coeficiente PIB/Frijol

Coeficiente Importaciones /Frijol

-.- Precio Frijol por mayor 
El segundo período, el cual se extiende desde 1969 hasta 1986, muestra la tendencia alcista del precio, como resultado, entre otras cosas, no de la presión de la acumulación, pues ésta no ha vuelto a tener repunte como el de los años sesenta, sino de la contracción considerable de las importaciones desde 1969, después de la guerra con Honduras.

Debemos insistir en que el alza de los precios del frijol obedece a muchas causas, entre otras al alza de los costos de los insumos y del transporte, pero fundamentalmente es el resultado tendencial de la limitada producción interna y de la insuficiente complementación a través de importaciones por cuasa de nuestra restricción externa de balanza de pagos.

Conforme al gráfico 2, el cuadro medio ( $/ / \mathrm{Cw}, \mathrm{T})$ es representativo del comportamiento de nuestra economia en lo que se refiere al frijol durante los años sesenta: alta acumulación -déficit externo- estabilidad de precios. Por el contrario, el cuadrante izquierdo ( $/ / \mathrm{Cw}, \mathrm{Pw}$ ) es el representativo de los años setenta y ochenta: menor acumulación-inflación-estabilidad externa (frijol).

En lo que se refiere al comportamiento más reciente los cuadros II y III muestran la evolución comparada de los precios de los granos

\section{Cuadro II \\ Precios promedio en el primer semestre de granos básicos seleccionados}

( $₫$ /libra)
1987

0.48

1.25

1.10
1988

3.00

1.52

Fuente: Economía Agropecuaria, Ministerio de Agricultura y Ganaderia. 


\section{Cuadro III}

Evolución mensual durante 1988 de los precios de granos básicos seleccionados ( $₫ /$ libra).

Enero Febrero Marzo Abril Mayo Junio

$\begin{array}{lllllll}\text { maiz } & 0.50 & 0.50 & 0.50 & 0.50 & 0.60 & 0.60 \\ \text { frijol rojo } & 1.90 & 2.10 & 2.75 & 3.60 & 3.60 & 4.25 \\ \text { arroz } & 1.40 & 1.40 & 1.50 & 1.60 & 1.60 & 1.60\end{array}$

Fuente: IDEM.

básicos.

El primer semestre de 1988 ha sido especial en el comportamiento del precio del frijol. Como se ve en el cuadro II su precio promedio aumentó en 140\% entre el primer semestre de 1987 y el primer semestre de 1988. Asimismo el cuadro III nos muestra que este crecimiento se desató a partir de febrero. El impacto del crecimiento del precio del frijol sobre la inflación general del país se aprecia en el siguiente cuadro:

\section{Cuadro IV}

Variación mensual de índice de precios al consumidor en 1988 (año base 1978=100).

Enero Febrero Marzo Abril Mayo Junio Enero-Junio

\begin{tabular}{|l|l|l|l|l|l|l|l|}
\hline alimentos & 1.7 & 2.0 & 5.0 & 5.3 & 2.8 & 3.4 & 3.4 \\
vestuario & 0.4 & 0.1 & 0.2 & 0.6 & 1.0 & 0.6 & 0.5 \\
\hline vivienda & 0.2 & 0.1 & 0.1 & 0.1 & 0.9 & 0.3 & 0.3 \\
\hline miscelaneos & 4.4 & 1.8 & 0.1 & 1.2 & 1.3 & 1.2 & 1.1 \\
\hline general & 1.7 & 1.4 & 2.7 & 2.8 & 2.0 & 2.2 & 2.1 \\
\hline
\end{tabular}

Fuente: MIPLAN.

No hay duda que la inflación reciente ha tenido un origen esencialmente agrícola y fue provocada por la contracción de la producción en el ciclo 1987-1988 resultado de la sequía del año recien pasado. Su impacto sobre la producción de frijol se desprende del cuadro comparativo siguiente: 


\section{Cuadro V}

Superficie y producción de frijol años 1987/1988 - 1988/1989a

$\begin{array}{cc}\begin{array}{c}\text { Superticie } \\ \text { (manzanas) }\end{array} & \begin{array}{c}\text { Producción } \\ \text { (quintales) }\end{array} \\ 87 / 88-88 / 89 & 87 / 88-88 / 89 \\ 89,300 \quad 82,100 & 531,000 \quad 903,000\end{array}$

Fuente: Ministerio de Agricultura y Ganadería.

a) intención de siembra.

Los datos anteriores muestran que aunque en este próximo ciclo se intentará sembrar una superticie inferior a la que efectivamente se sembró durante el periodo anterior, se espera que la produción casi duplique a la del ciclo 87/88, debido a las buenas lluvias de 1988. Esta comparación es suficiente, pues, para percibir la contracción que nuestra producción de frijol experimentó el año anterior por causa de la sequía y la presión inflacionaria que desató.

\section{Conclusiones}

El resultado más importante que se desprende del análisis precedente es que la producción agricola de alimentos constituye un límite para la acumulación en el resto de la economía. Este límite, cuando es rebasado, da paso a presiones inflacionarias y/o a déficit en el balance externo alimentario. En otras palabras, podemos afirmar que dado el nivel de producción agrícola y un determinado crecimiento de la economia, existe un trade off entre déficit externo e inflación.

También ha quedado demostrado que dada cierta capacidad importadora existe una trayectoria de crecimiento no inflacionario, la cual supone un determinado crecimiento de la producción doméstica de alimentos. Asimismo, este límite de origen agricola del crecimiento estable se expande con mayor capacidad importadora, la cual se alcanza con mayores exportaciones.

Asi, pues, el crecimiento de la economía para ser estable requiere, en consecuencia, de una adecuada planificación de la expansión de la producción agrícola de alimentos y de las exportaciones de otros bienes.

La evidencia empírica nos ha mostrado que la estabilidad de precios de los alimentos durante los años sesenta estuvo asociada, en el caso 
del frijol, a la complementación de nuesta producción doméstica con suficientes importaciones. La caída de las importaciones a partir de 1969 y el estancamiento de la producción de alimentos ha sometido a la sociedad a una presión inflacionaria de origen agrícola, la cual no ha sido la única que ha estado actuando en estos años, pero que permanentemente ha estado presente. Esta presión inflacionaria básica se ha acentuado en distintos momentos, tal es el caso durante el primer semestre de 1988 causado por la sequia del año anterior.

Los desarrollos y resultado que aquí hemos obtenido deben llevarnos a comprender que los procesos inflacionarios no sólo son los resultados de las expansiones globales de la demanda provocados por el crecimiento del dinero, sino también los procesos a través de los cuales se ajusta la economía ante los desbalances intersectoriales y, por lo tanto, no sólo fenómenos monetarios sino también reales, más perceptibles, como señalara Juan Noyola, en los paises subdesarrollados.

\section{Citas}

- Departamento de Economía, Universidad Centroamericana José Simeón Cañas, San Salvador.

1. Noyola, J. (1984), pg. 353.

2. Ibid, pg. 354.

3. El papel del grado de la organización sindical en el crecimiento de los precios fue destacado desde 1776 por Adam Smith (1984): "El operario desea sacar lo más posible y los patrones dar lo menos que puedan. Sin embargo, no es difícil de prever cual de las dos partes saldrá gananciosa en la disputa.... los patrones, siendo menos en número, se pueden poner de acuerdo más fácilmente, además de que las leyes autorizan sus asociaciones o, por lo menos, no las prohiben, mientras que, en el caso de los trabajadores, las desautorizan" pg. 65.

4. La asociación entre grado de monopolio y margen de ganancia fue establecido formalmente por Kalecki (1982).

5. Noyola, J. lbid, pg. 354.

6. Vease Kalecki (1983) y Kalecki (1982).

\section{Bibliografia}

1. Kalecki Michal (1982), "Ensayos escogidos sobre dinámica de la economla capitalista", México, D. F., Fondo de Cultura Económica.

2. Kalecki, Michal (1983), "Las ecuaciones marxistas de reproducción y la economía moderna", México, D. F., Investigación Económica, número 166, Octubre-Noviembre.

3. Llon, M. y Trigueros, A. (1988), "Productividad agroalimentaria", San Salvador, Tesis de Licenciatura, Universidad Centroamerica José Simeón Cañas. 
4. Marx, Karl (1976), " El capital". Tomo II. México, D. F., Siglo XXI.

5. Noyola, Juan (1984), "El desarrollo económico y la inflación en México y otros países latinoamericanos". México, D. F., Investigación Económica, núm. 169, Julio -Septiembre.

6. Rivera C., Roberto (1988), "La inflación en El Salvador". Revista de la Integración y el Desarrollo de Centro América, Núm, 41, Banco Centroamericano de Integración Económica.

7. Smith, Adam (1984), "Investigación sobre la naturaleza y causas de la riqueza de las naciones ", México, D. F., Fondo de Cultura Económica.

Digitalizado por Biblioteca "P. Florentino Idoate, S.J." Universidad Centroamericana José Simeón Cañas 